\title{
Measuring thermophysical properties by IR thermography
}

by $P$. Bison

CNR-ITC, C.so Stati Uniti 4, 35127 Padova, Italy - paolo.bison@itc.cnr.it

\section{Abstract}

Some thermographic techniques to measure thermophysical properties of solids are presented. The solutions of the heat transfer equation implied in the described techniques are discussed. Data analysis and uncertainties relations complement the description.

\section{Introduction}

It is important to know the thermal properties of materials in transient regime, to optimize the processes in which the flow of heat is relevant. For example, in the generation of the electric power, the materials are attentively selected from the point of view of the thermal properties. Also, in the automotive industry where braking systems should dissipate large amount of heat produced by the friction, thermal conductivity is a crucial parameter. The same is true in aeronautics where heat shields are used. The thermal properties acting in transient regime are thermal inertia and thermal diffusivity. Thermal inertia, a.k.a. effusivity is the property of the material that measures the reaction to the delivery of a certain amount of energy with a temperature variation. The greater the thermal inertia the lower the temperature variation. By definition the thermal inertia is

$$
e=\sqrt{\lambda \rho c_{p}}
$$

where $\lambda$ is thermal conductivity, $\rho$ is density and $c_{p}$ is specific heat. Thermal inertia is expressed in SI units as $\left[\mathrm{Jm}^{-2} \mathrm{~K}^{-1} \mathrm{~s}^{-1 / 2}\right]$. That implies measurements of energy, space, temperature and time. Comparing thermal inertia with thermal diffusivity

$$
\alpha=\frac{\lambda}{\rho c_{p}}
$$

whose SI units are $\left[\mathrm{m}^{2} \mathrm{~s}^{-1}\right]$ we notice how the latter needs only space and time measurements, being in that respect a simpler quantity to evaluate. It is obvious that the knowledge of both these quantities gives the thermal conductivity

$$
\lambda=\sqrt{\alpha} \cdot e
$$

and the heat capacity

$$
\rho c_{p}=\frac{e}{\sqrt{\alpha}}
$$

of the material under test. Thermal inertia is a parameter that plays its role in the transient heating and cooling of bodies. From this point of view it differs from the heat capacity in so far the latter is measured in equilibrium conditions, while the former take into account also the ability of the body to diffuse the heat (via the thermal conductivity) not only to store it.

Thermal inertia and thermal diffusivity are preferably measured in transient conditions by imposing a heat flux on the surface of the material, that in time is described by an impulsive, modulated or step function. A common way to do so is using special lamps whose electromagnetic flux is transformed in heat, once absorbed by the surfaces of the opaque bodies. Such transient techniques that utilizes light sources are categorized as photothermal. It is common to evaluate the transient temperature of the bodies by IR detector, minimizing any perturbation of the measured surface. Suitable models give finally the result.

The work is subdivided in these parts:

1) presentation of the heat equation heating)

2) solutions that depends linearly on the parameters: the semi-infinite body (pulse, step and modulated

3) data reduction, effusivity and diffusivity identification, by linear least-squares method, uncertainties evaluation 
4) examples: building materials and metals

5) solutions that depends non-linearly on the parameters: the slab and the two layers system (pulse and modulated heating)

6) data reduction, effusivity and diffusivity identification, by non-linear least-squares method, uncertainty evaluation.

7) examples on thermal barrier coating (TBC)

8) parameters anisotropy: solutions for localized heat source

9) 2D data reduction by spatial Fourier Transform analysis and linear least-squares method, uncertainty evaluation.

10) examples on TBC 